\title{
Safety and efficacy of endoscopic resection for the treatment of duodenal subepithelial lesions
}

\author{
Chen $\mathrm{Li}^{1,2}$, Yi Chu ${ }^{1,2}$, Liang $\mathrm{Lv}^{1,2}$, Xuehong Wang ${ }^{1,2}$, Meihong Yu ${ }^{1,2}$, Yuyong Tan ${ }^{1,2}$, Deliang Liu ${ }^{1,2}$ \\ ${ }^{1}$ Department of Gastroenterology, The Second Xiangya Hospital, Central South University, Changsha, China; ${ }^{2}$ Research Center of Digestive \\ Disease, Central South University, Changsha, China \\ Contributions: (I) Conception and design: D Liu, Y Tan; (II) Administrative support: None; (III) Provision of study materials or patients: None; (IV) \\ Collection and assembly of data: C Li; (V) Data analysis and interpretation: All authors; (VI) Manuscript writing: All authors; (VII) Final approval of \\ manuscript: All authors. \\ Correspondence to: Yuyong Tan; Deliang Liu. Department of Gastroenterology, The Second Xiangya Hospital, Central South University, Changsha \\ 410011, China; Research Center of Digestive Disease, Central South University, Changsha 410011, China. \\ Email: tanyuyong@csu.edu.cn; deliangliu@csu.edu.cn.
}

\begin{abstract}
Background: Endoscopic management of duodenal subepithelial lesions is challenging, and there are only a few studies on this topic. This study aimed to evaluate the safety and efficacy of endoscopic resection for the treatment of duodenal subepithelial lesions.

Methods: We retrospectively analyzed the clinical data, including epidemiologic characteristics, therapeutic outcomes, complications, and follow-up results, of 49 patients with duodenal subepithelial lesions who underwent endoscopic resection at our hospital between August 2010 and September 2019.

Results: We performed 35 endoscopic submucosal dissection, 9 endoscopic mucosal resection, 3 endoscopic submucosal excavation, and 2 endoscopic full-thickness resection. The en bloc resection rate and R0 resection rate were $95.9 \%$ and $89.8 \%$, respectively. Delayed perforations developed in 2 (4.1\%) patients; surgical intervention was required for both. Coagulation syndrome developed in 1 (2.0\%) patient; however, it was treated conservatively. Delayed bleeding or other serious complications did not occur. One patient underwent complementary surgery after endoscopic resection. One (2.3\%) recurrence occurred in patients who underwent endoscopic resection at a median follow-up duration of 24 months (range, 1-88 months).

Conclusions: Endoscopic resection is an effective, safe, and minimally invasive method for the histopathologic assessment and curative treatment of duodenal subepithelial lesions originating from the submucosal or muscularis propria.
\end{abstract}

Keywords: Endoscopic resection; duodenal subepithelial lesions (D-SELs); therapeutic outcomes; efficacy; safety

Submitted Jul 27, 2020. Accepted for publication Dec 07, 2020.

doi: 10.21037/jgo-20-301

View this article at: http://dx.doi.org/10.21037/jgo-20-301

\section{Introduction}

Duodenal subepithelial lesions (D-SELs) are mostly asymptomatic lesions that are incidentally detected during endoscopic examinations. Most D-SELs are benign, including Brunner's gland hyperplasia, lipomas, and ectopic pancreases; however, some are malignant or have malignant tendencies, such as neuroendocrine neoplasms (NENs) and gastrointestinal stromal tumors (GISTs) (1). The initial management of SELs depends on the accurate diagnosis and evaluation of the malignant potential of the lesions. Biopsy samples are obtained with difficulty due to the subepithelial location of the lesions. Endoscopic ultrasonography (EUS) is the most helpful imaging technique for evaluating SELs. EUS-guided fine-needle aspiration can be beneficial in some cases $(2,3)$. However, the final diagnosis can only be obtained by performing tumor resection (4).

There is controversy regarding the optimal treatment 




Figure 1 Flow diagram based on the results of endoscopic resection for duodenal SELs. SELs, subepithelial lesions; ESD, endoscopic submucosal dissection; EMR, endoscopic mucosal resection; ESE, endoscopic submucosal excavation; EFTR, endoscopic full-thickness resection.

strategy for D-SELs. Surgery, including limited resection and pancreaticoduodenectomy, is invasive, expensive, and involves a high risk of severe complications, such as hemorrhage, pancreatic fistula, and intra-abdominal infection (5). With the recent rapid development of endoscopic techniques and devices, endoscopic resection (ER) has become an alternative method of diagnosing and treating D-SELs. The main ER types are endoscopic mucosal resection (EMR), endoscopic submucosal dissection (ESD), endoscopic submucosal excavation (ESE), and endoscopic full-thickness resection (EFTR) (6-8). However, ER for D-SELs remains challenging and is associated with a high incidence of complications because of the narrow cavity, thin wall, and large vascular network of the duodenum. Only a few studies on the efficacy and safety of ER for the treatment of duodenal lesions are available, and these studies rarely focused on D-SELs. Therefore, we aimed to perform a retrospective study of 49 patients with SELs in the duodenum to evaluate the efficacy and safety of ER for the treatment of D-SELs $(6,7,9)$.

We present the following article in accordance with the STROBE reporting checklist. Available at http://dx.doi. org/10.21037/jgo-20-301.

\section{Methods}

\section{Patients}

We retrospectively analyzed the clinical data of patients with D-SELs who underwent ER at the Second Xiangya Hospital between August 2010 and September 2019 (Figure 1). All D-SELs included in this study originated from the submucosal or muscularis propria (MP) layer, as ascertained by EUS (U-ME1 or A75; Olympus, Tokyo, Japan) before the procedure. The indications for ER were as follows: (I) D-SELs suspected or confirmed to be potentially malignant tumors with no risk of metastases by preoperative EUS, abdominal computed tomography (CT), and/or histopathology; (II) symptomatic SELs; (III) SELs increasing rapidly in size during the followup period; or (IV) a strong desire to undergo ER. The exclusion criteria were as follows: (I) signs of metastases or invasion outside the duodenum; (II) imaging features indicating high risk, such as poorly defined margins and heterogeneity; (III) periampullary lesions; (IV) inability to tolerate the procedure; and $(\mathrm{V})$ severe coagulation disorders. Written informed consent was obtained from the patients before endoscopic treatment. The study was 

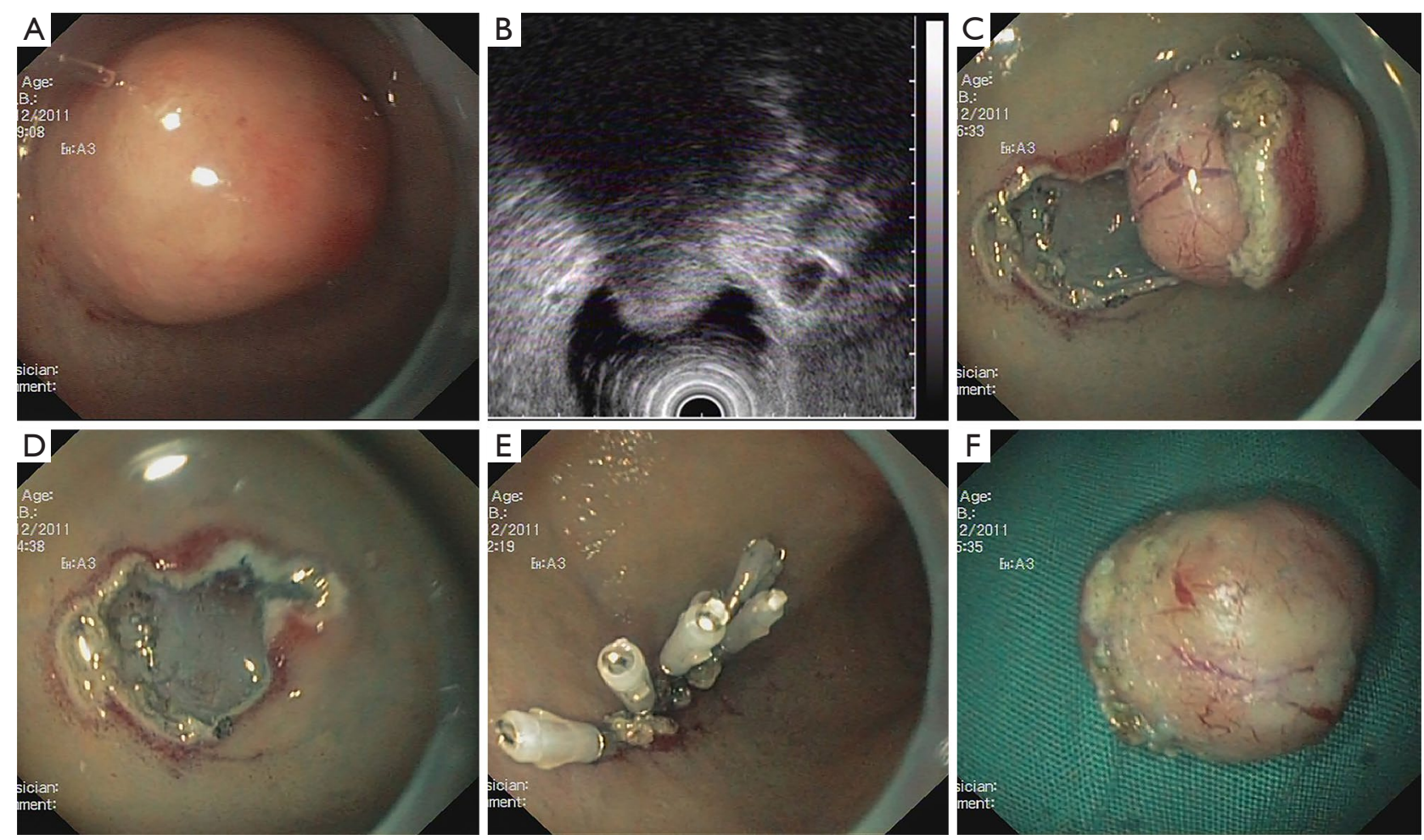

Figure 2 Endoscopic submucosal dissection of a Brunner's gland hyperplasia. (A) Endoscopic view of the subepithelial lesion located in the duodenal bulb; (B) the tumor originated from the submucosal layer on EUS; (C) submucosal dissection; (D) wound surface after tumor removal; (E) wound closure with clips; (F) the resected specimen. EUS, endoscopic ultrasonography.

conducted in accordance with the Declaration of Helsinki (as revised in 2013). The study protocol was approved by the Ethics Review Board of the Second Xiangya Hospital (No. LYF2020129).

\section{ER procedures}

Thirty-six (73.5\%) ER procedures were performed with patients under general anesthesia, while 13 (26.5\%) were performed with patients under conscious sedation. ER was performed with a single-channel endoscope (GIFQ260J; Olympus) with an attached transparent cap (D201-11802; Olympus). Other equipment included an argon plasma coagulation unit (APC300; ERBE; Tübingen, Germany), a high-frequency generator (ICC 200, ICC 300, or VIO 200D; ERBE), a carbon dioxide insufflator (UCR; Olympus), an injection needle (NM-4L-1; Olympus), hot biopsy forceps (FD-1U-1; Olympus), a dual knife (KD650Q; Olympus), an insulation-tip knife (KD-611L; Olympus), and hemostatic clips (HX-600-90; Olympus). During EMR, a solution $(100 \mathrm{~mL}$ normal saline, $1 \mathrm{~mL}$ indigo carmine, and $1 \mathrm{~mL}$ epinephrine) was injected into the submucosal layer. Subsequently, the lesion was resected using a snare. For ESD (Figure 2) and ESE, dots were marked around the lesion using a dual knife (KD-650Q; Olympus), followed by administration of a submucosal injection. After precutting the mucosa and submucosa, the tumor was dissected (ESD) or excavated as deep as the MP layer around the lesion (ESE) using a dual knife or insulation-tip knife. Thereafter, the mucosal defect was closed using metal clips or an endoloop combined with metal clips, as necessary. The EFTR procedure was performed as reported previously (10) (Figure 3). All resected specimens were embedded in paraffin and then sectioned for pathological assessment.

\section{Postoperative management}

For all patients, proton pump inhibitors, antibiotics, and hemostatics were administered intravenously for 3 days; then, oral proton pump inhibitors were prescribed for 1 month. Patients who underwent ESD, EMR, or ESE 


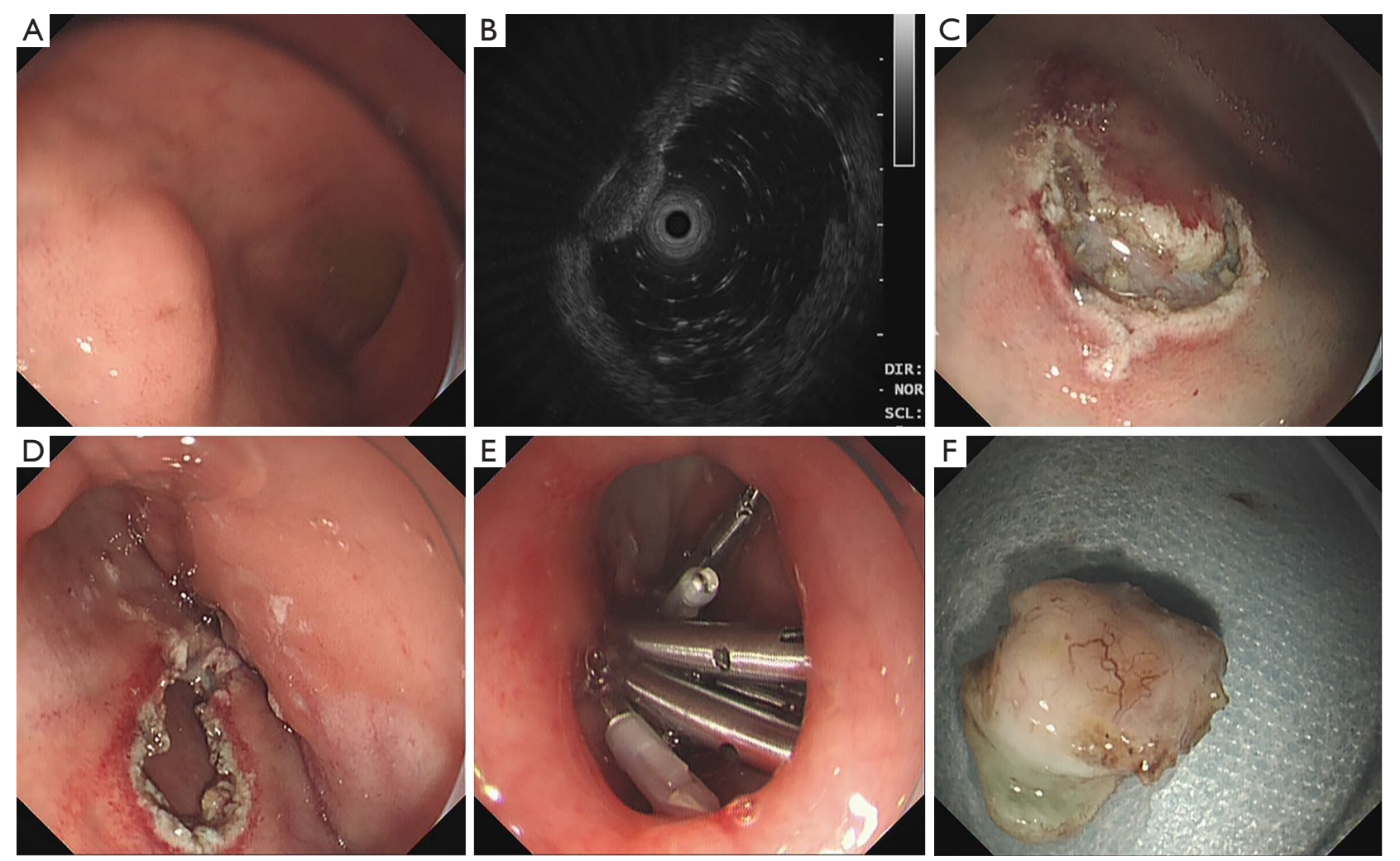

Figure 3 Endoscopic full-thickness resection of an ectopic pancreas. (A) Endoscopic view of the subepithelial lesion located in the duodenal bulb; (B) the tumor originated from the muscularis propria layer on EUS; (C) tumor resection; (D) duodenal wall defect after tumor removal; (E) wound closure with clips; (F) the resected specimen. EUS, endoscopic ultrasonography.

were kept nil per os for the first 24 to $48 \mathrm{~h}$. Thereafter, they were allowed to consume a liquid diet for 3-5 days and were gradually allowed to return to a normal diet. Patients who underwent EFTR were kept nil per os for the first $72 \mathrm{~h}$. Thereafter, they were allowed to consume a liquid diet for 5 days. On postoperative day 4, contrast roentgenography was performed to check for any leakage.

\section{Outcome parameters}

The main outcome parameters in this study were en bloc resection rate, $\mathrm{R} 0$ resection rate, delayed perforation rate, delayed bleeding rate, and recurrence rate. The procedure time, hospital stay duration, and cost were assessed as secondary outcomes.

$E n$ bloc resection was defined as resection of the lesion in one piece. R0 resection was defined as en bloc resection with tumor-free horizontal and vertical margins. Intraprocedural bleeding was defined as persistent bleeding that could not be managed by endoscopic methods and transfusion or other interventions were required during the procedure. Bleeding that occurred during the procedure and was controlled endoscopically was not considered intra-procedural bleeding. Delayed bleeding was defined as hematemesis, melena, or a decrease of more than $2 \mathrm{~g} / \mathrm{dL}$ in the hemoglobin level after the procedure. Intraprocedural perforation was considered if an endoscopic view of an extra-duodenal structure was observed during the procedure. Delayed perforation was defined as a sudden onset of abdominal pain with retroperitoneal pneumatosis or free gas detected on CT scans or abdominal radiographs after resection of the lesion. Intentional perforation was not considered a complication of EFTR. Coagulation syndrome was considered when abdominal pain developed with or without fever and no sign of free gas was observed using abdominal imaging after the procedure $(11,12)$. The costs included all the expenses incurred during hospitalization, which included the cost of preoperative assessment, procedure-related, and post-operative management. 


\begin{tabular}{lc}
$\begin{array}{l}\text { Table } 1 \text { Characteristics of } 49 \text { patients who underwent endoscopic } \\
\text { resection for duodenal subepithelial lesions }\end{array}$ & Value \\
\hline Characteristics & $1.5(0.4-7.0)$ \\
\hline Median tumor size, cm (range) & $31(63.3)$ \\
Tumor size, $\mathrm{n}(\%)$ & $18(36.7)$ \\
$<2 \mathrm{~cm}$ & \\
$\geq 2 \mathrm{~cm}$ & $37(75.5)$ \\
Tumor location, $\mathrm{n}(\%)$ & $6(12.2)$ \\
Bulb & $6(12.2)$ \\
Descending portion & \\
Junction of the first and second portions & $42(85.7)$ \\
Layer of tumor origin, $\mathrm{n}(\%)$ & $7(14.3)$ \\
Submucosa & \\
Muscularis propria & $39(79.6)$ \\
Macroscopic type, $\mathrm{n}(\%)$ & $10(20.4)$ \\
Sessile & \\
Pedunculated &
\end{tabular}

\section{Follow-up}

Surveillance endoscopy or EUS with or without CT was performed at 1, 6, and 12 months postoperatively and annually thereafter to observe wound healing and check for residual tumors and metastases. Patients lost to follow-up were excluded when calculating the follow-up parameters such as recurrence rate, follow-up duration, etc.

\section{Statistical analysis}

SPSS 23.0 software (SPSS Inc., Chicago, IL, USA) was used for statistical analyses. The categorical variables were represented as frequencies and analyzed using the chisquare test or Fisher's exact test. The continuous variables were expressed as mean \pm standard deviation or median and range and calculated using the Student's $t$-test or Mann-Whitney $\mathrm{U}$ test; $\mathrm{P}<0.05$ was considered statistically significant.

\section{Results}

\section{Characteristics of patients and SELs}

All patients (24 men and 25 women; average age, $52.0 \pm 11.8$ years; range, $26-72$ years) underwent successful
Table 2 Postoperative histology for duodenal subepithelial lesions

\begin{tabular}{lc}
\hline Histology & $\mathrm{N}(\%)$ \\
\hline Brunner's gland hyperplasia & $20(40.8)$ \\
Neuroendocrine neoplasms & $8(16.3)$ \\
Lipoma & $8(16.3)$ \\
Ectopic pancreas & $6(12.2)$ \\
GIST & $2(4.1)$ \\
Solitary Peutz-Jeghers polyp & $1(2.0)$ \\
Inflammatory fibroid polyp & $1(2.0)$ \\
Brunner gland cystadenoma & $1(2.0)$ \\
Fibroma & $1(2.0)$ \\
Gangliocytoma & $1(2.0)$ \\
\hline
\end{tabular}

GIST, gastrointestinal stromal tumor.

ER. A total of 49 lesions were resected successfully. Their characteristics are summarized in Table 1. The median diameter of the lesions was $1.5 \mathrm{~cm}$ (range, $0.4-7.0 \mathrm{~cm}$ ). The average diameter of NENs was $1.1 \pm 0.5 \mathrm{~cm}$ (range, $0.5-2.0 \mathrm{~cm})$. Thirty-seven $(75.5 \%)$ lesions were located in the bulb, $6(12.2 \%)$ in the descending portion, and 6 $(12.2 \%)$ at the junction of the first and second portions of the duodenum.

\section{Histology}

Table 2 shows the postoperative histology. Forty-two (85.7\%) lesions, including Brunner's gland hyperplasia $(\mathrm{n}=18)$, NENs [n=8, including 2 cases of G1 neuroendocrine tumors (NETs), 5 cases of G2 NETs, and 1 case of neuroendocrine carcinoma which was $0.5 \mathrm{~cm}$ in diameter and confined to the submucosa], ectopic pancreas $(n=2)$, lipomas $(n=8)$, GIST ( $n=1$, very low risk category based on the modified National Institutes of Health criteria (13)], solitary PeutzJeghers polyp $(\mathrm{n}=1)$, inflammatory fibroid polyp $(\mathrm{n}=1)$, Brunner gland cystadenoma $(n=1)$, fibroma $(n=1)$, and gangliocytoma $(n=1)$, were from the submucosal layer. Seven $(14.3 \%)$ lesions including ectopic pancreas $(n=4)$; Brunner's gland hyperplasia $(\mathrm{n}=2)$; and GIST $(\mathrm{n}=1$, very low risk category), were from the MP. No cancerous changes were seen in the 20 Brunner's gland hyperplasia lesions.

\section{Outcomes of ER}

ESD, EMR, ESE, and EFTR were performed for 35 
Table 3 Therapeutic outcomes of endoscopic resection for duodenal subepithelial lesions

\begin{tabular}{lc}
\hline Therapeutic outcomes & Value \\
\hline En bloc resection, $\mathrm{n}(\%)$ & $47(95.9)$ \\
Ro resection, $\mathrm{n}(\%)$ & $44(89.8)$ \\
Procedure time, minutes [range] & $45[15-155]$ \\
Complication, $\mathrm{n}(\%)$ & $3(6.1)$ \\
Delayed perforation & $2(4.1)$ \\
Coagulated syndrome & $1(2.0)$ \\
Recurrence, $\mathrm{n}(\%)$ & $1(2.3)$ \\
Hospital stay, days [range] & $7[3-45]$ \\
\hline
\end{tabular}

(71.4\%), $9(18.4 \%), 3(6.1 \%)$, and $2(4.1 \%)$ lesions, respectively. The therapeutic outcomes of ER are summarized in Table 3. The overall en bloc resection rate was 95.9\% [47/49]. Two patients underwent EMR using the piecemeal technique (one with Brunner's gland hyperplasia and the other with lipoma). The $\mathrm{R} 0$ resection rate was $89.8 \%$ [44/49]. Positive vertical margins were detected in 2 patients (one with G1 NET and the other with G2 NET) with ESD, and they refused additional treatment. No recurrence was noticed during the follow-up period of 8 and 23 months, respectively. In another case of G1 NET, the normal tissue covering the tumor was damaged during $\mathrm{ER}$, and this resection was classified as non-R0 resection. The en bloc resection rate for ESD $(100 \%, 35 / 35)$ was higher than that of EMR $(77.8 \%, 7 / 9, \mathrm{P}<0.05)$; however, the R0 resection rates for ESD and EMR were 91.4\% [32/35] and 77.8\% [7/9], respectively, with no significant difference $(\mathrm{P}>0.05)$. Patients who underwent EMR incurred lower costs $(\mathrm{P}<0.05)$ than those who underwent ESD (Table 4). The median procedure time was $45 \mathrm{~min}$ (range, $15-155 \mathrm{~min})$. The median hospital stay duration was 7 days (range, 3-45 days).

\section{Complications}

Two (4.1\%) patients, one with a $1.5-\mathrm{cm}$ lipoma resected by EMR and the other with a 2.0-cm NET (G2) resected by ESD, developed delayed perforation; both tumors arising from the submucosa. Both patients recovered after open surgical closure of duodenal perforation. Coagulation syndrome occurred in $1(2.0 \%)$ patient who underwent ESD; however, the patient recovered conservatively. Delayed bleeding, gas-related complications, or duodenal
Table 4 Comparison of therapeutic outcomes between ESD and EMR

\begin{tabular}{lccc}
\hline Therapeutic outcomes & ESD & EMR & $P$ \\
\hline Tumor size, cm (range) & $1.6(0.4-7.0)$ & $1.5(0.5-4.0)$ & 0.482 \\
En bloc resection, $\mathrm{n}(\%)$ & $35(100.0)$ & $7(77.8)$ & 0.038 \\
R0 resection, $\mathrm{n}(\%)$ & $32(91.4)$ & $7(77.8)$ & 0.267 \\
Complication, $\mathrm{n}(\%)$ & $2(5.7)$ & $1(11.1)$ & 0.506 \\
Delayed perforation & $1(2.9)$ & $1(11.1)$ & 0.371 \\
Coagulation syndrome & $1(2.9)$ & $0(0)$ & 1.000 \\
Procedure time, min [range] & $40[15-155]$ & $30[15-60]$ & 0.066 \\
Hospital stay, day [range] & $6[3-14]$ & $7[3-45]$ & 0.790 \\
Cost, USD (range) & $3,096.4$ & $2,057.2$ & 0.011 \\
& $(1,769.6-$ & $(1,165.7-$ & \\
& $9,889.2)$ & $11,795.6)$ & \\
Local recurrence, $\mathrm{n}(\%)$ & $1(3.1)$ & $0(0)$ & 1.000 \\
\hline ESD, endoscopic submucosal dissection; EMR endoscopic \\
ucosal resection.
\end{tabular}

stricture was not observed in our study.

\section{Follow-up}

Of 49 patients, four were lost to follow-up and one with a G2 NET underwent additional surgery because of tumor infiltration to the MP layer. Follow-up data were available for $44(89.8 \%)$ patients. The median follow-up period was 24 months (range, 1-88 months). One (2.3\%) case of tumor recurrence was noted in a patient with Brunner's gland hyperplasia at 42 months after ESD. The recurring tumor was resected using laparoscopic local resection. The two cases of GISTs were followed-up for 10 and 47 months, respectively, without recurrence. The patient who underwent R0 resection of a neuroendocrine carcinoma refused to undergo additional treatment; no recurrence occurred after 8 months of follow-up.

\section{Discussion}

The endoscopic management of D-SELs is challenging, and there are only a few studies on the efficacy and safety of endoscopic management. Our study reported that ER is an effective treatment for D-SELs associated with low complication rates and low recurrence risk.

The overall en bloc resection rate and $\mathrm{R} 0$ resection rate were $95.9 \%$ and $89.8 \%$, respectively. The en bloc resection 
rate for ESD was higher than that for EMR, which is in accordance with the results of previous studies on duodenal epithelial tumors $(14,15)$. The R0 resection rates for ESD was higher than EMR. However, the difference was not statistically significant (91.4\% versus $77.8 \%, \mathrm{P}>0.05)$. However, these results should be interpreted with caution due to the small sample size.

Bleeding and perforation are the most common complications of ER. Delayed perforation developed in 2 patients in this study, resulting in a perforation rate of $4.1 \%$, which was consistent with previous studies $(6,7,16,17)$. The reported incidence of bleeding for duodenal ER ranges from $0 \%$ to $14.9 \%(6,7,14,17)$, and no delayed bleeding was observed in this study.

The indications for different ER methods for the treatment of D-SELs have not been standardized to date. The selection of ER methods is associated with the lesion features, such as macroscopic type, size, origin, growth pattern, and so on, which are ascertained by preoperative evaluation, including white light endoscopy, EUS, and/or CT. At our center, EMR is suitable for small $(<2 \mathrm{~cm})$, intraluminally growing SELs arising from the muscularis mucosa or superficial submucosa that can be removed in one piece using a snare. When lesions with intraluminal growth are confined to the deep submucosa or superficial MP layer, or cannot be resected in one piece by a snare ( $>2 \mathrm{~cm}), \mathrm{ESD}$ is preferred. If SELs arise from the deep MP layer, then ESE is recommended. EFTR is optimal for SELs growing extraluminally or those that are tightly connected to the serosa.

All duodenal NENs have a potential for malignancy (18). ER is recommended for duodenal NENs that are $\leq 1 \mathrm{~cm}$ in size, non-ampullary, confined to the submucosal layer, and do not present with lymph node or distant metastasis (19). Eight NENs with an average diameter of $1.1 \pm 0.5 \mathrm{~cm}$ (range, $0.5-2.0 \mathrm{~cm}$ ) were included in this study. All lesions were located in the bulb, originated from the submucosa, and were resected by ESD. The R0 resection rate for ESD of NENs in the present study was low $(62.5 \%, 5 / 8)$, which may be related to the insufficiency of EUS for defining the lesion type. The differential diagnoses for hypoechoic and heterogeneous lesions from the submucosal and MP layers may be broad, such as GISTs, leiomyomas, and NENs, and EUS-guided fine-needle aspiration may be useful for determining the malignant potential of these lesions to guide further management (2). Therefore, for D-SELs $>1 \mathrm{~cm}$ in size that are suspected to be or confirmed as NENs preoperatively, therapeutic management should be selected with caution.
Although our study showed that ER was effective and safe and that the long-term prognosis associated with ER was good, there are several limitations of this study. First, bias may potentially be present mainly because of the singlecenter retrospective design and relatively small sample size. Second, all procedures were performed by experienced endoscopists at a tertiary hospital; therefore, the results cannot be directly generalized. Third, a small number of EFTR and ESE procedures were performed owing to the endoscopist's concerns regarding perforation or bleeding during the selection of the management strategy. Due to these limitations, the results of our study should be interpreted carefully.

In summary, ER is an effective, safe, and minimally invasive method for the histopathologic assessment and curative treatment of D-SELs originating from the submucosal or MP layer and can serve as a promising alternative treatment for D-SELs. Its en bloc resection rate and $\mathrm{R} 0$ resection rate are high, and its recurrence rate and complication rate are low. Therefore, largescale, randomized, controlled, and multicenter studies are warranted to confirm the efficacy and safety of ER for D-SELs.

\section{Acknowledgments}

We would like to thank the pathologists form our hospital for the critical pathological diagnosis.

Funding: None.

\section{Footnote}

Reporting Checklist: The authors have completed the STROBE reporting checklist. Available at http://dx.doi. org/10.21037/jgo-20-301

Data Sharing Statement: Available at http://dx.doi. org/10.21037/jgo-20-301

Conflicts of Interest: All authors have completed the ICMJE uniform disclosure form (Available at http://dx.doi. org/10.21037/jgo-20-301). The authors have no conflicts of interest to declare.

Ethical Statement: The authors are accountable for all aspects of the work in ensuring that questions related to the accuracy or integrity of any part of the work are appropriately investigated and resolved. Written informed 
consent was obtained from the patients before endoscopic treatment. The study was conducted in accordance with the Declaration of Helsinki (as revised in 2013). The study protocol was approved by the Ethics Review Board of the Second Xiangya Hospital (No. LYF2020129).

Open Access Statement: This is an Open Access article distributed in accordance with the Creative Commons Attribution-NonCommercial-NoDerivs 4.0 International License (CC BY-NC-ND 4.0), which permits the noncommercial replication and distribution of the article with the strict proviso that no changes or edits are made and the original work is properly cited (including links to both the formal publication through the relevant DOI and the license). See: https://creativecommons.org/licenses/by-nc-nd/4.0/.

\section{References}

1. Cho JW; Korean ESD Study Group. Current Guidelines in the Management of Upper Gastrointestinal Subepithelial Tumors. Clin Endosc 2016;49:235-40.

2. Standards of Practice Committee, Faulx AL, Kothari S, et al. The role of endoscopy in subepithelial lesions of the GI tract. Gastrointest Endosc 2017;85:1117-32.

3. Turhan N, Aydog G, Ozin Y, et al. Endoscopic ultrasonography-guided fine-needle aspiration for diagnosing upper gastrointestinal submucosal lesions: a prospective study of 50 cases. Diagn Cytopathol 2011;39:808-17.

4. Longcroft-Wheaton G, Bhandari P. Endoscopic resection of submucosal tumors. Expert Rev Gastroenterol Hepatol 2015;9:659-69.

5. Lee SY, Goh BK, Sadot E, et al. Surgical Strategy and Outcomes in Duodenal Gastrointestinal Stromal Tumor. Ann Surg Oncol 2017;24:202-10.

6. Kim TW, Kim GH, Park DY, et al. Endoscopic resection for duodenal subepithelial tumors: a single-center experience. Surg Endosc 2017;31:1936-46.

7. Ye LP, Mao XL, Zheng HH, et al. Safety of endoscopic resection for duodenal subepithelial lesions with wound closure using clips and an endoloop: an analysis of 68 cases. Surg Endosc 2017;31:1070-7.

8. Cai MY, Martin Carreras-Presas F, Zhou PH. Endoscopic full-thickness resection for gastrointestinal submucosal tumors. Dig Endosc 2018;30 Suppl 1:17-24.

9. Oono Y, Shinmura K, Hori K, et al. Endoscopic submucosal resection using a ligation device without injection for duodenal neuroendocrine tumors. Surg Endosc 2019;33:2008-14.

10. Tan Y, Tang X, Guo T, et al. Comparison between submucosal tunneling endoscopic resection and endoscopic full-thickness resection for gastric stromal tumors originating from the muscularis propria layer. Surg Endosc 2017;31:3376-82.

11. Hirasawa K, Sato C, Makazu M, et al. Coagulation syndrome: Delayed perforation after colorectal endoscopic treatments. World J Gastrointest Endosc 2015;7:1055-61.

12. Yamaguchi H, Fukuzawa M, Kawai T, et al. Predictive Factors of Postendoscopic Submucosal Dissection Electrocoagulation Syndrome and the Utility of Computed Tomography Scan after Esophageal Endoscopic Submucosal Dissection. Digestion 2020;101:579-89.

13. Joensuu H. Risk stratification of patients diagnosed with gastrointestinal stromal tumor. Hum Pathol 2008;39:1411-9.

14. Hoteya S, Furuhata T, Takahito T, et al. Endoscopic Submucosal Dissection and Endoscopic Mucosal Resection for Non-Ampullary Superficial Duodenal Tumor. Digestion 2017;95:36-42.

15. Yamamoto Y, Yoshizawa N, Tomida H, et al. Therapeutic outcomes of endoscopic resection for superficial nonampullary duodenal tumor. Dig Endosc 2014;26 Suppl 2:50-6.

16. Watanabe D, Hayashi H, Kataoka Y, et al. Efficacy and safety of endoscopic submucosal dissection for nonampullary duodenal polyps: A systematic review and metaanalysis. Dig Liver Dis 2019;51:774-81.

17. Kim GH, Kim JI, Jeon SW, et al. Endoscopic resection for duodenal carcinoid tumors: a multicenter, retrospective study. J Gastroenterol Hepatol 2014;29:318-24.

18. Sato Y, Hashimoto S, Mizuno K, et al. Management of gastric and duodenal neuroendocrine tumors. World J Gastroenterol 2016;22:6817-28.

19. Delle Fave G, O'Toole D, Sundin A, et al. ENETS Consensus Guidelines Update for Gastroduodenal Neuroendocrine Neoplasms. Neuroendocrinology 2016;103:119-24.

Cite this article as: $\mathrm{Li} \mathrm{C}$, Chu Y, Lv L, Wang X, Yu M, Tan Y, Liu D. Safety and efficacy of endoscopic resection for the treatment of duodenal subepithelial lesions. J Gastrointest Oncol 2021;12(2):856-863. doi: 10.21037/jgo-20-301 